\title{
A Geometrical Representation of Entanglement as Internal Constraint
}

\author{
Diederik Aerts, Ellie D'Hondt*and Bart D'Hooghe ${ }^{\dagger}$ \\ Centrum Leo Apostel (CLEA), Vrije Universiteit Brussel \\ Krijgskundestraat 33, B1160 Brussels, Belgium \\ diraerts,eldhondt,bdhooghe@vub.ac.be
}

\begin{abstract}
We study a system of two entangled spin $1 / 2$, were the spin's are represented by a sphere model developed within the hidden measurement approach which is a generalization of the Bloch sphere representation, such that also the measurements are represented. We show how an arbitrary tensor product state can be described in a complete way by a specific internal constraint between the ray or density states of the two spin $1 / 2$. We derive a geometrical view of entanglement as a 'rotation' and 'stretching' of the sphere representing the states of the second particle as measurements are performed on the first particle. In the case of the singlet state entanglement can be represented by a real physical constraint, namely by means of a rigid rod.
\end{abstract}

\section{Introduction}

Within the hidden measurement approach to quantum mechanics [1], 2] entanglement has been studied for a system consisting of two entangled spin $\frac{1}{2}$

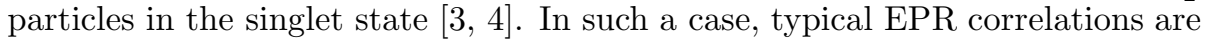
encountered, meaning that if one of both spins collapses in a certain direction under the influence of a measurement, then the other spin collapses in the opposite direction. In [9, 10] these results were generalized to give a description of entanglement as a hidden correlation between the proper states of the individual subsystems. Our aim is to elaborate on these results, more specifically, we want to develop a geometrical representation of entanglement by means of an internal constraint between the states of the spin $1 / 2$ particles, represented on the sphere, for an arbitrary tensor product state that is not necessarily the singlet state. We do this by introducing constraint functions, which describe the behavior of the state of one of the spins if measurements are executed on

\footnotetext{
${ }^{*}$ Research Assistant of the Fund for Scientific Research - Flanders (Belgium)

${ }^{\dagger}$ Postdoctoral Fellow of the Fund for Scientific Research - Flanders (Belgium)
} 
the other spin. In 四 the internal constraint was given a real physical classical mechanics representation, namely by means of a rigid rod. An interesting question remained whether it is also possible for non singlet states to invent a similar mechanistic device.

For the individual spin $1 / 2$ entities we use a sphere model representation developed within the hidden measurement approach to quantum mechanics [1, 2, 3, 5, 6, 7, 8, which is a generalization of the Bloch or Pauli representation, such that also the measurements are represented. We identify a parameter $r \in[0,1]$, arising from the Schmidt diagonal decomposition, that is a measure of the amount of entanglement, such that for $r=0$ the system is in the singlet state with maximal entanglement (and we recover previous results), and for $r=1$ the system is in a pure product state. For intermediate values of $r$ we encounter new situations in which entanglement is expressed by a rotation and distortion of the sphere, representing the state of the single spin $1 / 2$ entities.

Concerning measurements and their effect on one of the spins in an entangled state when executed on the other spin, we consider on the one hand a measurement on a pure state followed by a collapse of the state, as prescribed by Von Neumann's formula, and on the other hand a measurement on a mixed state resulting in a new mixed state, as prescribed by Luder's formula. We will show that an arbitrary collapse measurement on one spin provokes a rotation and a stretching on the other spin, which can be described in detail by means of the sphere model, and an arbitrary measurement on one of the two spins in a density state does not provoke any change in the partial trace density matrix of the other spin, i.e., the spins behave as separated entities for such measurements.

\section{The Sphere Model}

The sphere model is a generalization of the Bloch sphere representation, such that also the measurements as well as a parameter for non-determinism can be represented [8]. In this model, a spin $1 / 2$ state $|\psi\rangle=\left(\cos \frac{\theta}{2} e^{\frac{-i \phi}{2}}, \sin \frac{\theta}{2} e^{\frac{i \phi}{2}}\right)$ is represented by the point $u(1, \theta, \phi)=(\sin \theta \cos \phi, \sin \theta \sin \phi, \cos \theta)$ on the surface of a 3-dimensional unit sphere, often called Bloch or Poincaré sphere. All points of the Bloch sphere represent states of the spin, such that points on the surface correspond to pure states, while interior points correspond to density states. This is because an arbitrary point $u(r, \theta, \phi), r \in[0,1], \theta \in[0, \pi], \phi \in[0,2 \pi]$, of the Bloch sphere can in general be written as a convex linear combination $u(r, \theta, \phi)=r u(1, \theta, \phi)+(1-r) u(0, \theta, \phi)$ from which follows the corresponding density state

$$
D(r, \theta, \phi)=r D(1, \theta, \phi)+(1-r) D(0, \theta, \phi)=\frac{1}{2}\left(\begin{array}{cc}
1+r \cos \theta & r \sin \theta e^{-i \phi} \\
r \sin \theta e^{i \phi} & 1-r \cos \theta
\end{array}\right)
$$

In this expression $D(1, \theta, \phi)=|\psi\rangle\langle\psi|$ is the usual density state representation of a pure state, while $D(0, \theta, \phi)$ is the density matrix representing the center of the sphere (the singlet state). Next to this, the sphere model allows a representation 
of measurements. Without loss of generality we can demonstrate the effect of such a measurement by considering states that are on the straight line connecting the North pole $D(1,0, \phi)=|0\rangle\langle 0|$ and the South pole $D(1, \pi, \phi)=|1\rangle\langle 1|$ of the sphere (we use the convention that $|0\rangle$ corresponds to spin up or $(1,0)$, while $|1\rangle$ corresponds to spin down or $(0,1))$. In this case, the spin is in density state $D(r, 0,0)$. After a measurement of the spin in the direction $u(1, \theta, \phi)$, the density state of the spin becomes (by means of Luder's Formula)

$$
D=P(\theta, \phi) D(r, 0,0) P(\theta, \phi)+(1-P(\theta, \phi)) D(r, 0,0)(1-P(\theta, \phi))
$$

where $P(\theta, \phi)$ is the projector on the ray state $|\theta \phi\rangle$, and hence equals $D(1, \theta, \phi)$. For $\theta \in\left[0, \frac{\pi}{2}\right]$, this results in the density matrix

$$
D=\frac{1}{2}\left(\begin{array}{cc}
1+r^{\prime} \cos \theta & r^{\prime} \sin \theta e^{-i \phi} \\
r^{\prime} \sin \theta e^{i \phi} & 1-r^{\prime} \cos \theta
\end{array}\right)=D\left(r^{\prime}, \theta, \phi\right)
$$

where $r^{\prime}=r \cos \theta$. A similar expression $D\left(r^{\prime}, \theta^{\prime}, \phi^{\prime}\right)$, with $r^{\prime}=r \cos \theta^{\prime}, \theta^{\prime}=\pi-\theta$ and $\phi^{\prime}=\phi+\pi$, is obtained for $\theta \in\left[\frac{\pi}{2}, \pi\right]$. If we consider the sphere we can see easily that in both cases the point $u(r, 0,0)$ is transformed into the point

$$
(u(r, 0,0) \cdot u(1, \theta, \phi)) u(1, \theta, \phi)
$$

This means that we have identified a very simple mechanics to describe the quantum measurement effect on a mixed state in our sphere model. The effect is just an ordinary orthogonal projection on the direction of the spin measurement of the point that represents the density state of the spin in the sphere model, as represented in Fig. 1.

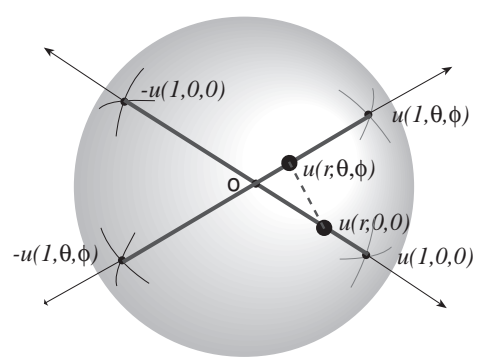

Figure 1: Effect of the measurement on a single spin $\frac{1}{2}$.

In general, suppose that we have a spin state represented by the point $u(s, \alpha, \beta)$ and we perform a spin measurement in direction $(\theta, \phi)$. If we denote the orthogonal projection on the direction $(\theta, \phi)$ by $E(\theta, \phi)$, the new state after this measurement is given by

$$
\left\{\begin{array}{l}
E(\theta, \phi) u(s, \alpha, \beta)=u(s \cos \theta, \theta, \phi) \text { if }|\alpha-\theta| \in\left[0, \frac{\pi}{2}\right] \\
E(\theta, \phi) u(s, \alpha, \beta)=u(s \cos (\pi-\theta), \pi-\theta, \phi+\pi) \text { if }|\alpha-\theta| \in\left[\frac{\pi}{2}, \pi\right]
\end{array}\right.
$$

It is possible to give a nice geometrical presentation of how the spin state changes under the influence of measurements in different directions, as shown in Fig. 2. 


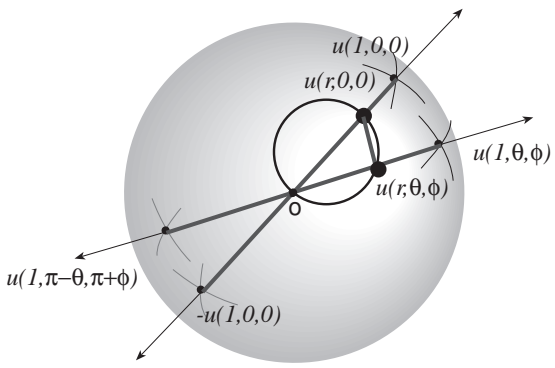

Figure 2: A geometrical presentation of how the spin state changes under the influence of measurements in different directions.

More concretely, consider a little sphere with as North pole the point $u(s, \alpha, \beta)$, the point that represents the spin state, and as South pole the center of the big sphere of the model. The spin state is transformed to the point of intersection between this little sphere and the direction of the measurement performed. Hence the points of the little sphere are those points representing the states where the spin state can be transformed to, under arbitrary angles of measurement.

\section{Constraint Functions}

A system of two entangled spin $\frac{1}{2}$ is described by means of an arbitrary unit vector $|\psi\rangle \in \mathbb{C}_{1}^{2} \otimes \mathbb{C}_{2}^{2}$, in which $\mathbb{C}_{1}^{2}$ and $\mathbb{C}_{2}^{2}$ are two copies of $\mathbb{C}^{2}$, which we label with indices 1 and 2 with the sole purpose of identifying them. The vector $|\psi\rangle$ can always be written as the following linear combination $|\psi\rangle=\sum_{i j} \lambda_{i j}\left|e_{1}^{i}\right\rangle \otimes\left|e_{2}^{j}\right\rangle$ where $\lambda_{i j} \in \mathbb{C}$, and $\left\{\left|e_{1}^{i}\right\rangle\right\}$ and $\left\{\left|e_{2}^{j}\right\rangle\right\}$ are bases of $\mathbb{C}_{1}^{2}$ and $\mathbb{C}_{2}^{2}$ respectively. When we carry out a collapse measurement on the first spin, Von Neumann's formula describes how it collapses into a spin state described by the unit vector $\left|x_{1}\right\rangle \in \mathbb{C}_{1}^{2}$, thus transforming the entangled state $|\psi\rangle$ into $\left(P_{\left|x_{1}\right\rangle} \otimes I\right)(|\psi\rangle)$ where $P_{\left|x_{1}\right\rangle}$ is the orthogonal projector on $\left|x_{1}\right\rangle$ in $\mathbb{C}_{1}^{2}$, and $I$ is the unit operator in $\mathbb{C}_{2}^{2}$. The result is that the entangled spins end up in the following product state $\left|x_{1}\right\rangle \otimes \sum_{i j} \lambda_{i j}\left\langle x_{1}, e_{1}^{i}\right\rangle\left|e_{2}^{j}\right\rangle$. This means that as a consequence of the measurement on the first spin, collapsing its state to $\left|x_{1}\right\rangle$, the second spin collapses to the state $\sum_{i j} \lambda_{i j}\left\langle x_{1}, e_{1}^{i}\right\rangle\left|e_{2}^{j}\right\rangle$.

In an analogous way we can show that if a measurement is performed on the second spin, resulting in a collapse to the state $x_{2} \in \mathbb{C}_{2}^{2}$, the state of the first spin becomes $\sum_{i j} \lambda_{i j}\left\langle x_{2}, e_{2}^{j}\right\rangle\left|e_{1}^{i}\right\rangle$. Because of this, we arrive at the following definition.

Definition 1 (Constraint Functions). We define the constraint functions 
$F_{12}(\psi)$ and $F_{21}(\psi)$ related to $\psi$ in the following way

$$
\begin{aligned}
& F_{12}(\psi) \quad: \quad \mathbb{C}_{1}^{2} \rightarrow \mathbb{C}_{2}^{2}:\left|x_{1}\right\rangle \mapsto \sum_{i j} \lambda_{i j}\left\langle x_{1}, e_{1}^{i}\right\rangle\left|e_{2}^{j}\right\rangle \\
& F_{21}(\psi): \mathbb{C}_{2}^{2} \rightarrow \mathbb{C}_{1}^{2}:\left|x_{2}\right\rangle \mapsto \sum_{i j} \lambda_{i j}\left\langle x_{2}, e_{2}^{j}\right\rangle\left|e_{1}^{i}\right\rangle
\end{aligned}
$$

In other words, the constraint functions map the state where one of the spins collapses to by a measurement to the state that the other spin collapses to under influence of the entanglement correlation. A detailed study of the constraint functions can give us a complete picture of how the entanglement correlation works as an internal constraint. Before we arrive at this complete picture, however, we give some properties of the constraint functions.

One can show that the following properties hold for the constraint functions and the relation between the two constraint functions $F_{12}(\psi)$ and $F_{21}(\psi)$ :

Proposition 1. The constraint functions are canonically defined.

Proposition 2. The constraint functions are conjugate linear.

Proposition 3. $\left\{\begin{array}{l}D_{1}(\psi) \equiv t r_{\mathbb{C}_{1}^{2}}|\psi\rangle\langle\psi|=F_{21}(\psi) \circ F_{12}(\psi) \\ D_{2}(\psi) \equiv t r_{\mathbb{C}_{2}^{2}}|\psi\rangle\langle\psi|=F_{12}(\psi) \circ F_{21}(\psi)\end{array}\right.$ or in other words,

$F_{21}(\psi) \circ F_{12}(\psi)$ equals $D_{1}(\psi)$, i.e., the partial trace density matrix over $\mathbb{C}_{2}^{2}$ and $F_{12}(\psi) \circ F_{21}(\psi)$ equals $D_{2}(\psi)$, i.e., the partial trace density matrix over $\mathbb{C}_{1}^{2}$.

Proposition 4. For $\left|x_{1}\right\rangle \in \mathbb{C}_{1}^{2}$ and $\left|x_{2}\right\rangle \in \mathbb{C}_{2}^{2}$ we have

$$
\left\langle F_{12}(\psi)\left(\left|x_{1}\right\rangle\right), x_{2}\right\rangle=\left\langle x_{1}, F_{21}(\psi)\left(\left|x_{2}\right\rangle\right)\right\rangle^{*}
$$

To derive a complete view of how entanglement works as an internal constraint for a 2-particle system, we now work out the relation between the Schmidt diagonal form (e.g. 11]) and the constraint functions. We begin by choosing the base $\left|x_{1}^{1}\right\rangle=\left(\cos \frac{\theta}{2} e^{-i \frac{\phi}{2}}, \sin \frac{\theta}{2} e^{\frac{i \phi}{2}}\right),\left|x_{1}^{2}\right\rangle=\left(-i \sin \frac{\theta}{2} e^{-i \frac{\phi}{2}}, i \cos \frac{\theta}{2} e^{\frac{i \phi}{2}}\right)$ in $\mathbb{C}_{1}^{2}$. With respect to this basis, expression (1) for a general density matrix becomes

$$
D_{1}(\psi)=\frac{1}{2}\left(\begin{array}{cc}
1+r & 0 \\
0 & 1-r
\end{array}\right)
$$

One can choose a basis $\left\{\left|x_{2}^{1}\right\rangle,\left|x_{2}^{2}\right\rangle\right\}$ in $\mathbb{C}_{2}^{2}$ given by

$$
\left|x_{2}^{1}\right\rangle=\frac{\sqrt{2}}{\sqrt{1+r}} F_{12}(\psi)\left(\left|x_{1}^{1}\right\rangle\right),\left|x_{2}^{2}\right\rangle=\frac{\sqrt{2}}{\sqrt{1-r}} F_{12}(\psi)\left(\left|x_{1}^{2}\right\rangle\right)
$$

One can show that $\left\|x_{2}^{1}\right\|^{2}=1=\left\|x_{2}^{2}\right\|^{2}$ and

$$
D_{2}(\psi)\left(\left|x_{2}^{1}\right\rangle\right)=\frac{1+r}{2}\left|x_{2}^{1}\right\rangle, D_{2}(\psi)\left(\left|x_{2}^{2}\right\rangle\right)=\frac{1-r}{2}\left|x_{2}^{2}\right\rangle
$$


Hence $\left|x_{2}^{1}\right\rangle$ and $\left|x_{2}^{2}\right\rangle$ are normalized eigenvectors of $D_{2}(\psi)$ with eigenvalues $\frac{1+r}{2}$ and $\frac{1-r}{2}$ respectively. Therefore, with respect to the basis $\left\{\left|x_{2}^{1}\right\rangle,\left|x_{2}^{2}\right\rangle\right\}, D_{2}(\psi)$ is expressed as

$$
D_{2}(\psi)=\frac{1}{2}\left(\begin{array}{cc}
1+r & 0 \\
0 & 1-r
\end{array}\right)
$$

Finally, let us find the expression for $\psi$ with respect to the basis $\left\{\left|x_{1}^{1}\right\rangle \otimes\right.$ $\left.\left|x_{2}^{1}\right\rangle,\left|x_{1}^{1}\right\rangle \otimes\left|x_{2}^{2}\right\rangle,\left|x_{1}^{2}\right\rangle \otimes\left|x_{2}^{1}\right\rangle,\left|x_{1}^{2}\right\rangle \otimes\left|x_{2}^{2}\right\rangle\right\}$ of $\mathbb{C}_{1}^{2} \otimes \mathbb{C}_{2}^{2}$. In general, this expression is of the form $\psi=a\left|x_{1}^{1}\right\rangle \otimes\left|x_{2}^{1}\right\rangle+b\left|x_{1}^{1}\right\rangle \otimes\left|x_{2}^{2}\right\rangle+c\left|x_{1}^{2}\right\rangle \otimes\left|x_{2}^{1}\right\rangle+d\left|x_{1}^{2}\right\rangle \otimes\left|x_{2}^{2}\right\rangle$. However, since

$$
\begin{aligned}
& F_{12}(\psi)\left(\left|x_{1}^{1}\right\rangle\right)=a\left|x_{2}^{1}\right\rangle+b\left|x_{2}^{2}\right\rangle=\frac{\sqrt{1+r}}{\sqrt{2}}\left|x_{2}^{1}\right\rangle \\
& F_{12}(\psi)\left(\left|x_{1}^{2}\right\rangle\right)=c\left|x_{2}^{1}\right\rangle+d\left|x_{2}^{2}\right\rangle=\frac{\sqrt{1-r}}{\sqrt{2}}\left|x_{2}^{2}\right\rangle
\end{aligned}
$$

we obtain

$$
a=\frac{\sqrt{1+r}}{\sqrt{2}}, b=0, c=0, d=\frac{\sqrt{1-r}}{\sqrt{2}}
$$

Thus, the Schmidt diagonal form of $|\psi\rangle$ is given by

$$
|\psi\rangle=\frac{\sqrt{1+r}}{\sqrt{2}}\left|x_{1}^{1}\right\rangle \otimes\left|x_{2}^{1}\right\rangle+\frac{\sqrt{1-r}}{\sqrt{2}}\left|x_{1}^{2}\right\rangle \otimes\left|x_{2}^{2}\right\rangle
$$

\section{Measurements}

With all the above we can now concentrate on the role of measurements. More particularly, we analyze how a measurement, carried out on one subentity of an entangled system, affects the state of the other subentity of which the entangled system is composed. We discuss both the effect of a measurement on density states as described by Luder's formula and the effect of a collapse measurement on a pure state as described by Von Neumann's formula.

To describe the effect of a measurement on the density state of a subsystem we use Luder's formula (2), where in this case the initial density state is calculated from the Schmidt diagonal form (16) derived above. Choosing bases $\left\{x_{1}^{1}=(1,0), x_{1}^{2}=(0,1)\right\}$ in $\mathbb{C}_{1}^{2}$ and $\left\{x_{2}^{1}=(1,0), x_{2}^{2}=(0,1)\right\}$ in $\mathbb{C}_{2}^{2}$, one can calculate the density state $D(\psi)=|\psi\rangle\langle\psi|$ corresponding with the pure state $|\psi\rangle$. After the measurement this state has changed into the density state $D^{\prime}(\psi)$ given by Luder's formula: $D^{\prime}(\psi)=(P(\theta, \phi) \otimes \mathbf{1}) D(\psi)(P(\theta, \phi) \otimes \mathbf{1})+$ $((\mathbf{1}-P(\theta, \phi)) \otimes \mathbf{1}) D(\psi)((\mathbf{1}-P(\theta, \phi)) \otimes \mathbf{1})$, from which we can calculate $D_{1}(\psi)$, i.e., the partial trace density matrix to $\mathbb{C}_{1}^{2}$, obtaining

$$
D_{1}(\psi)=\frac{1}{2}\left(\begin{array}{cc}
1+r \cos ^{2} \theta & r \sin \theta \cos \theta e^{-i \phi} \\
r \sin \theta \cos \theta e^{i \phi} & 1-r \cos ^{2} \theta
\end{array}\right)
$$

This is the same density matrix as we found in expression (3), i.e. after carrying out a measurement on a single spin $\frac{1}{2}$ in a density state. On the other hand, if 
we calculate $D_{2}(\psi)$, i.e., the partial trace density matrix to $\mathbb{C}_{2}^{2}$, we find:

$$
D_{2}(\psi)=\frac{1}{2}\left(\begin{array}{ll}
1+r & 0 \\
0 & 1-r
\end{array}\right)
$$

which is independent of $(\theta, \phi)$. From expressions (17) and $(\sqrt{18})$, one derives that a measurement prescribed by Luder's formula on one spin does not provoke any change in the partial trace density matrix of the other spin: in other words, the spins behave as separated entities for such measurements.

Let us now study what happens when a collapse measurement is performed on one of the subsystems in the entangled system. Since the constraint functions describe exactly this, studying collapse measurements means studying the constraint function, more specifically how they map points of the sphere (i.e., quantum states) onto one another. As a point of departure, we choose $\psi$ and bases as in the above, where both bases are connected through equation (10). From these equations, we first observe that within the sphere model, they imply that the north (south) pole of the first sphere is mapped onto the north (south) pole of the second sphere. Next, it follows immediately that $F_{12}(\psi)$ does not conserve the norm. Indeed, the norm of $F_{12}(\psi)(|x\rangle)$ for an arbitrary vector $|x\rangle=x(\theta, \phi)$ is as follows:

$$
\| F_{12}(\psi)(|x\rangle) \|^{2}=\frac{1+r}{2} \cos ^{2} \frac{\theta}{2}+\frac{1-r}{2} \sin ^{2} \frac{\theta}{2}=\frac{1}{2}(1+r \cos \theta)
$$

If we consider for a moment the angle $\theta$ as a variable, we see that the square of the norm varies between $\frac{1+r}{2}$ and $\frac{1-r}{2}$, for the north $(\theta=0)$ and the south $(\theta=\pi)$ pole of the sphere respectively. Actually, this is where the factors $\sqrt{\frac{2}{1+r}}$ and $\sqrt{\frac{2}{1-r}}$ in the original definition of $\left|x_{2}^{1}\right\rangle$ and $\left|x_{2}^{2}\right\rangle$ in equation (10) come from. Not only the norm, but also orthogonality is in general not conserved by $F_{12}(\psi)$. For example, using the conjugate linearity of the constraint functions, we find that the two orthonormal vectors $\left|\psi_{u}\right\rangle=\psi(\theta, \phi)$ and $\left|\psi_{-u}\right\rangle=\psi(\pi-\theta, \phi+\pi)$ are mapped to

$$
\begin{aligned}
F_{12}(\psi)\left(\psi_{u}\right) & =\sqrt{\frac{1+r}{2}} \cos \frac{\theta}{2} e^{i \frac{\phi}{2}} x_{2}^{1}+\sqrt{\frac{1-r}{2}} \sin \frac{\theta}{2} e^{-i \frac{\phi}{2}} x_{2}^{2} \\
F_{12}(\psi)\left(\psi_{-u}\right) & =\sqrt{\frac{1+r}{2}} i \sin \frac{\theta}{2} e^{i \frac{\phi}{2}} x_{2}^{1}-i \sqrt{\frac{1-r}{2}} \cos \frac{\theta}{2} e^{-i \frac{\phi}{2}} x_{2}^{2}
\end{aligned}
$$

For $0 \neq \theta \neq \pi$ orthogonality is conserved if $\left\langle F_{12}(\psi)\left(\left|\psi_{u}\right\rangle\right), F_{12}(\psi)\left(\left|\psi_{-u}\right\rangle\right)\right\rangle=0$ with means that $r=0$. Translated on the sphere this means that diametrical opposite points are mapped to diametrical opposite points only in the special case $r=0$ (except for the north and south pole, which are always mapped onto the north and south pole of the second sphere). In other words, orthogonality only is generally conserved for the singlet state. While the norm and orthogonality are in general not conserved, we can look at the normalized image 
corresponding to a state $|x\rangle=x\left(\theta_{1}, \phi_{1}\right)$. In other words, we would like to know where the state

$$
|y\rangle=y\left(\theta_{2}, \phi_{2}\right)=\frac{1}{\| F_{12}(\psi)(|x\rangle) \|} F_{12}(\psi)(|x\rangle)
$$

lies on the sphere. Therefore, we compare corresponding inproducts on both spheres, and we obtain that

$$
\left\langle y, x_{2}^{1}\right\rangle=\sqrt{\frac{1+r}{1+r \cos \theta_{1}}} \cdot\left\langle x, x_{1}^{1}\right\rangle^{*}
$$

Again, we see that only for the singlet state inproducts are equal (and consequently, antipodal points on the sphere are mapped to antipodal points, as mentioned above). An interesting case is to look at the image of the equator, or in other words the points for which $\theta_{1}=\frac{\pi}{2}$. In this case

$$
\left\langle y, x_{2}^{1}\right\rangle=\sqrt{1+r} \cdot\left\langle x, x_{1}^{1}\right\rangle^{*}=\sqrt{1+r} \frac{1}{\sqrt{2}} e^{-i \frac{\phi_{1}}{2}}
$$

Translating this to the sphere model, using the following formula which expresses the relation between the inproduct of two vectors in $\mathbb{C}_{2}^{2}$ and the scalar product of the corresponding points in the sphere representation:

$$
\frac{1+\psi\left(\theta^{\prime}, \phi^{\prime}\right) \cdot \psi(\theta, \phi)}{2}=\left|\left\langle\psi\left(\theta^{\prime}, \phi^{\prime}\right), \psi(\theta, \phi)\right\rangle\right|^{2}
$$

applied to $|y\rangle$ and $\left|x_{2}^{1}\right\rangle$, we obtain

$$
\frac{1+y\left(\theta_{2}, \phi_{2}\right) \cdot x_{2}^{1}(\theta, \phi)}{2}=\frac{1+r}{2}
$$

and as a consequence: $y\left(\theta_{2}, \phi_{2}\right) \cdot x_{2}^{1}(\theta, \phi)=r$. This means that on the sphere, the elements of the equator are mapped onto a cone that makes an angle $\beta$ with the north south axis of the second sphere, such that $\cos \beta=r$. Once more, only for $r=0$ this is again an equator, hence conserving the angle between the elements of the equator and the north pole. For $r \in] 0,1[$ we obtain a cone with an angle $0<\beta<\frac{\pi}{2}$, which means that the equator has 'raised' to the north. For $r$ approaching 1 the sphere is stretched more and more to the north pole of the second sphere. Remember that in this limit case the superposition state becomes a product state, and this fits with the fact that for product states indeed the map $F_{12}(\psi)$ maps the first element of the product to the second. To see the general scheme we use equation (23), which yields

$$
y\left(\theta_{2}, \phi_{2}\right) \cdot x_{2}^{1}(\theta, \phi)=\frac{r+\cos \theta_{1}}{1+r \cos \theta_{1}}
$$

From this result it follows that straight lines through the center of the left sphere are mapped onto straight lines through the point $u(r, 0,0)$ along the north south axis in the second sphere. This gives a nice geometrical representation of this 
'stretching' on the second sphere, as shown in Fig. 3. Again, this shows that indeed only for the singlet state antipodal points of the first sphere are mapped onto antipodal points of the second sphere.
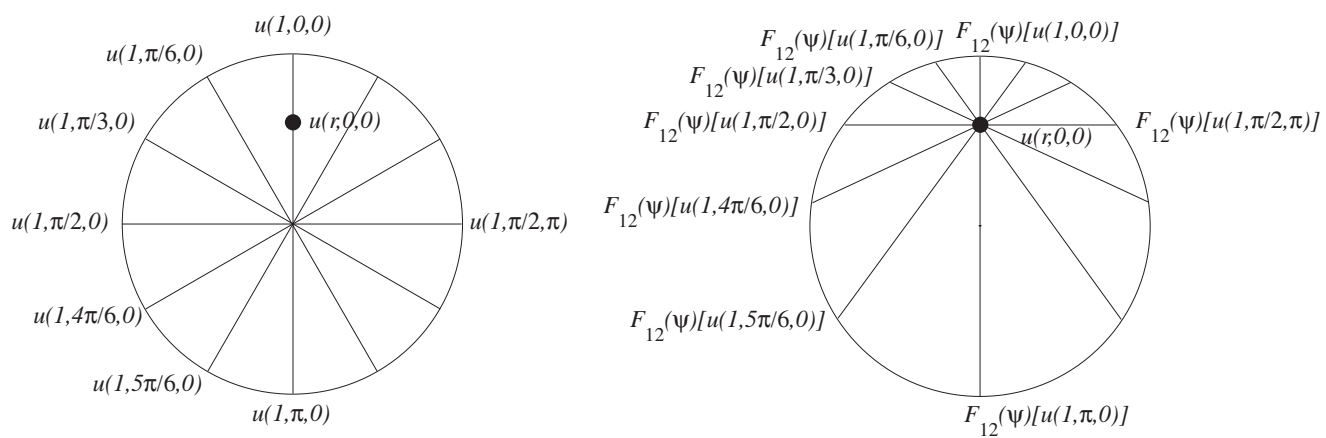

Figure 3: Straight lines through the center of the left sphere are mapped onto straight lines through the point $u(r, 0,0)$ along the north south axis in the second sphere.

\section{Conclusions}

We elaborated a formalism to model entanglement as an internal constraint. More specifically, we show that two spin $1 / 2$ particles in a nonproduct state can be described in a complete way by extracting entanglement into an internal constraint between the states of the particles. We introduce constraint functions, which describe the behavior of the state of one of the spins if measurements are executed on the other spin. In this way we can substitute the nonproduct state by the states of the individual particles and the internal constraint function. We make use of the sphere model representation for the spin's that was developed in Brussels, allowing for an easy to grasp visual support for the developed formalism. In deriving the effect measurements on one spin of an entangled state have on the other one, we differentiated between two types of measurements: measurements, of which the action on a mixture of states is described by Luder's formula, and collapse measurements, of which the action is described by Von Neumann's formula. Our result is that (1) an arbitrary Luder's measurement on one spin in a mixed state does not provoke any change in the partial trace density matrix of the other spin, i.e., the spins behave as separated entities for such measurements; (2) an arbitrary collapse measurement on one spin provokes a rotation and a stretching on the other spin, which gives a nice geometrical representation of how entanglement works as an internal constraint. The singlet state appears as a very special case in which norm and orthogonality are conserved. This makes it easier to understand that for the singlet state a real physical apparatus modelling the internal constraint can be built, namely a rigid rod connecting the two spins. Since for non singlet states norm and orthogonality are not conserved, and the geometrical representation entails rotation and more importantly stretching of the sphere, it is not obvious 
that a simple machinery (e.g. with a rigid rod) can be constructed in this case.

\section{References}

[1] Aerts, D., 1986, A possible explanation for the probabilities of quantum mechanics, J. Math. Phys., 27, 202-210.

[2] Aerts, D., 1987, The origin of the non-classical character of the quantum probability model, in Information, Complexity, and Control in Quantum Physics, eds. Blanquiere, A., Diner, S. and Lochak, G., Springer-Verlag, Wien-New York, 77-100.

[3] Aerts, D., 1991, A macroscopical classical laboratory situation with only macroscopical classical entities giving rise to a quantum mechanical probability model, in Quantum Probability and Related Topics, Volume VI, ed. Accardi, L., World Scientific Publishing Company, Singapore, 75-85.

[4] Aerts, D., 1991, A mechanistic classical laboratory situation violating the Bell inequalities with $2 \sqrt{2}$, exactly 'in the same way' as its violations by the EPR experiments, Helv. Phys. Acta, 64, 1-23.

[5] Aerts, D., Durt, T., 1994, Quantum, classical and intermediate, an illustrative example, Found. Phys., 24, 1353-1369.

[6] Aerts, D., Durt, T., 1994, Quantum, classical and intermediate: a measurement model, in Symposium on the Foundations of Modern Physics, eds. Laurikainen, K. V., Montonen, C. and Sunnaborg, K., Editions Frontieres, Gives Sur Yvettes, France.

[7] Aerts, D., Aerts, S., 1997, The hidden measurement formalism: quantum mechanics as a consequence of fluctuations on the measurement, in New Developments on Fundamental Problems in Quantum Physics, eds. Ferrero, M. and van der Merwe, A., Kluwer Academic, Dordrecht.

[8] Aerts, D., Aerts, S., Coecke, B., D'Hooghe, B., Durt, T. and Valckenborgh, F, 1997, A model with varying fluctuations in the measurement context, in New Developments on Fundamental Problems in Quantum Physics, eds. Ferrero, M. and van der Merwe, A., Kluwer Academic, Dordrecht.

[9] Coecke, B., 1998, A representation for compound systems as individual entities: hard acts of creation and hidden correlations, Found. Phys., 28, 1109.

[10] Coecke, B., 2000, Structural characterization of compoundness, Int. J. Theor. Phys. 39, 585.

[11] Nielsen, M. and Chuang, I., 2000, Quantum Computation and Quantum Information, Cambridge University Press. 